\title{
28 Research Square \\ TLR4 knock out alleviate both lung and liver inflammation caused by short-time exposure to PM2.5
}

Bijie Jiang ( $\nabla_{\text {jiangbijie1001@163.com ) }}$

Xinxiang Medical University https://orcid.org/0000-0002-2734-8109

Jianguo Lu

Xinxiang Medical University First Affiliated Hospital

Yang Liu

Xinxiang Medical University

Congmin Li

Centers for Disease Control and Prevention

Zhen An

Xinxiang Medical University

Yichun Bai

Xinxiang Medical University

Xinmei Zheng

Xinxiang Medical University

Weidong Wu

Xinxiang Medical University

\section{Research}

Keywords: TLR4, PM2.5, Lung, Liver, Inflammation

Posted Date: April 12th, 2021

DOl: https://doi.org/10.21203/rs.3.rs-388536/v1

License: (1) (1) This work is licensed under a Creative Commons Attribution 4.0 International License.

Read Full License 


\section{Abstract}

Background: Ambient particulate matter (PM), a principal component of air pollutant, is harmful to human health in many ways. Toll-like receptors (TLRs) are non-catalytic transmembrane protein that can identify conservative molecules from microorganisms. However, the regulatory mechanism of TLR4 and its effects on lung and liver inflammation induced by PM are unknown presently.

Method: Wild type mice and TLR4 ${ }^{-/-}$mice (C57BL/10JNju, mixed gender, 8-10 weeks old,), both were randomly divided into two groups. The mice were intratracheally instilled with saline or PM2.5 (4 mg/kg) every other day and continued for 10 days.

Result: PM2.5 increased the BCA and Lactate Dehydrogenase (LDH) level and TLR4 KO mice showed lower BCA compared to wild type mice $(P=0.08)$. PM2.5 treatment increased the expression of inflammatory cytokines (HMGB1, TNFa, MCP-1/CCL2, CCR2) significantly compared to the saline treatment $(P<0.05)$ and there is a trend that this could be attenuated in TLR4 knock out mice. HE staining showed that PM2.5 induced severe inflammatory damage, and TLR4 KO could alleviate the process of inflammation. As to the liver, LDH and Alkaline Phosphatase (AKP) significantly increased in PM2.5 treatment only in wild type mice. Similar to the lung tissue, PM2.5 stimulation resulted in robust induction of proinflammatory genes expression levels, and TLR4 KO could alleviate the progression of liver inflammation.

Conclusion: Our findings suggest that, TLR4 plays an important role in regulating lung and liver inflammatory response caused by PM2.5.

\section{Introduction}

Atmospheric pollutants are harmful to human health in many ways, mainly manifested by respiratory diseases and physiological dysfunction, as well as irritation of mucosal tissues such as eyes and nose ${ }^{1-}$ 3 . Recent studies have also shown that air pollutants can cause skin damage and skin aging through several pathways that induce oxidative stress, inflammation, apoptosis, and skin barrier dysfunction ${ }^{4-7}$.

The innate immune system serves as the first line of the host to defend against anonymous pathogenic invasion, relying on molecular determinant sensing of pathogen associated molecular patterns (PAMPs) 8,9 . The receptors that sense infections localized at various membrane compartments, including the plasma membrane, endosomes, and the endoplasmic reticulum ${ }^{10,11}$. TLRs are a class of pattern recognition receptors (PRRs) that include both intracellular and extracellular receptor families, and sense PAMPs or DAMPs (damage-associated molecular patterns). TLR signaling is complex and depends on other adapter protein and co-receptor pathway activation. Upon activation, TLRs then bind to specific adaptors to initiate the downstream signaling pathways leading to the production of inflammatory cytokines and chemokines. Past studies mostly focused on the intracellular TLRs (TLR3, TLR7, TLR9), 
but recent studies reveal that cell surface TLRs, especially TLR2 and TLR4, also play an essential role in the development of autoimmune diseases and afford multiple therapeutic targets ${ }^{12-15}$.

PM, a principal component of air pollutant, has been considered as the main contributor to haze weather 16,17. Particles less than $1 \mu \mathrm{m}$ in particulate matter deposit slowly and remain in the atmosphere for a long time. PM2.5 (particulate matter $\leq 2.5 \mu \mathrm{m}$ ) can be inhaled and deposited in human bronchi and alveoli, causing or aggravating respiratory diseases. Nevertheless, its mechanism has not been well explained, PM2.5 is recognized to exacerbate respiratory inflammation and cardiovascular diseases. It was reported recently that short- and long-term PM2.5 exposure increased plaque vulnerability via TLR4/MyD88/NF-KB pathway ${ }^{18}$. Numerous studies have focused on the harm of particulate matter to the respiratory system, however, more and more studies have found that PM2.5 can cause liver damage and liver fibrosis ${ }^{19-21}$. In the present study, the role of Toll-like receptor (TLR) 4 in exacerbation of lung and liver inflammation caused by urban PM2.5 was investigated. Our data together suggested that, TLR4 plays an important role in regulating lung and liver inflammatory response caused by PM2.5.

\section{Experimental Procedures}

\section{PM2.5 Collection and Preparation}

PM2.5 samples were collected onto quartz microfiber filters $(20.3 \times 25.4 \mathrm{~cm}, \mathrm{PALL}, \mathrm{USA})$ from Nov 2016 to Feb 2017, using a volumetric flow controlled system (TE-6070C, Tisch Environmental, USA) on the roof of the Research Building of Xinxiang Medical University. Each filter was weighted at least twice, and the difference of the values was less than or equal to $0.03 \mathrm{mg}$. The particles were eluted by ultrasonic processing for $15 \mathrm{~min}, 3$ times. Then freeze the eluent in a low-temperature refrigerator at $-80^{\circ} \mathrm{C}$. The samples were taken out 24 hours later and placed in a freeze dryer for freeze-drying treatment. When the water evaporates completely, grey dry flocs appeared at the bottom of the container. Store the collected PM2.5 dry samples in the refrigerator at $-20^{\circ} \mathrm{C}$. PM2.5 suspension $(2 \mathrm{mg} / \mathrm{ml})$ was prepared with sterile saline solution and stored at $4{ }^{\circ} \mathrm{C}$. Shake before use to make the particles evenly distributed.

\section{Animal Experiments}

Both male and female mice (Wild type or TIr4 ${ }^{\mathrm{lps}}$ - del, C57BL/10JNju) were purchased from Nanjing Model Animal Research Center. After rearing and reproducing to a certain number, then the formal experiments were set up.

Mice were housed in individual plastic cages under a 12/12 h light-dark cycle with free access to water and food at room temperature. All the animal experiments were conducted following the protocols approved by the University Committee on the Use and Care of Animals (UCUCA).

\section{Study Protocol}


Mice for the formal experiment (mixed gender, 8-10 weeks old), both wild type and TLR4 ${ }^{-/-}$, were randomly divided into two groups (4-5 mice per group). Inhalation tracheal drip method is used for dustcontamination. The mice were intratracheally instilled with saline or PM2.5 $(4 \mathrm{mg} / \mathrm{kg})$ every other day and continued for 10 days ( 5 times in total).

All the mice were sacrificed $48 \mathrm{~h}$ after the final intratracheal instillation. After anesthesia, eyeball blood was taken from mice, followed by BALF collection. Liver and lung portions were then removed and fixed with $4 \%$ paraformaldehyde solution. Some liver tissue and lung tissue were placed in liquid nitrogen and stored in $-80^{\circ} \mathrm{C}$. The weight of mice was also recorded before and after the experiment.

\section{Bronchoalveolar Lavage Fluid (BALF)}

A previously reported method was used to collect BALF. Briefly, after the blood collection, tracheas were cannulated. The left lung was lavaged by syringe with two injections of ice old sterile saline $(0.8 \mathrm{ml})$. The mean volume retrieved was $90 \%$ of the amount instilled $(1.6 \mathrm{ml})$. Fluids from the two lavages were then centrifuged at $1500 \mathrm{rpm}$ for $10 \mathrm{~min}$.

\section{Quantitative Real Time PCR (qPCR)}

Total RNAs were extracted using RNAiso Plus reagent (TaKaRa, 9770A). The first-strand cDNAs were synthesized using PrimeScript ${ }^{\text {TM }}$ RT Master Mix (TaKaRa, RR036A). Relative mRNA abundance of different genes was measured using TB Green ${ }^{\mathrm{TM}}$ Premix Ex Taq ${ }^{\mathrm{TM}}$ II (TaKaRa, RR820A) and ABI7500 real time PCR system (Applied Biosystems). qPCR Primers were designed as follows: HMGB1 forward, 5'GAAGTTGGACCCCAATGC-3', reverse, 5'-TCATCTGCTGCAGTGTTGTTCC-3'; MCP-1 forward, 5'ACTGAAGCCAGCTCTCTCTTCCTC-3', reverse, 5'-TTCCTTCTTGGGGTCAGCACAGAC-3'; TNFa forward, 5'CATCTTCTCAAAATTCGAGTGACAA-3', reverse, 5'-TGGGAGTAGACAAGGTACAACCC-3'; CCR2 forward, 5'CTC AGT TCA TCC ACG GCA TA-3', reverse, 5'- CAA GGC TCA CCA TCA TCG TA-3', 36B4 forward, 5'AAGCGCGTCCTGGCATTGTCT-3', reverse, 5'-CCGCAGGGGCAGCAGTGGT-3'. The expression levels of the target genes were quantified relative to the level of $36 \mathrm{~B} 4$ gene expression using the $2^{-\Delta \Delta C T}$ method. Realtime PCR experiments for each gene were performed on three separate occasions.

\section{HE staining}

Liver and lung tissues were fixed with $4 \%$ paraformaldehyde solution for at least $24 \mathrm{~h}$, and processed for routine paraffin histology. Paraffin sections were stained with hematoxylin and eosin using routine procedures. Finally, observe and take photos under a microscope.

\section{Statistical Analysis}

Data were presented as mean \pm S.E.M. Differences between groups were analyzed by Prism GraphPad 6 using Student's t-test (two-sided). ${ }^{\star} p<0.05, \# p<0.01$.

\section{Results}




\section{Bodyweight}

The process of PM2.5 infusion is a stress for mice, and improper operation is often accompanied by tongue damage. Therefore, we monitored the weight changes of mice during the whole experiment. We observed that there was downward trend from the third day, and most groups remained stable even though with slightly fluctuates (Fig. 1). However, mice challenged with PM2.5, wild type mice showed significantly declined compared to the TLR4 knock out mice (Fig. 1), and few mice developed severe morbidity or even death closely at the end of the experiment. This result revealed that PM2.5 decreased the bodyweight in WT mice, and this change was attenuated in TLR4 ${ }^{-/-}$mice.

\section{Lung inflammation}

To explore the pulmonary inflammation caused by PM2.5 in mice, alveolar lavage was performed in anesthetized mice. Bronchoalveolar lavage fluid (BALF) was collected and stored at $-20^{\circ} \mathrm{C}$. Total protein concentration and LDH were tested in a short time according to the instructions provided by the manufacturer. Total protein concentration was determined by BCA Protein Assy Kit (Tiangen Biotech, Beijing). LDH was determined by the LDH kit (Jiancheng Bioengineering Institute, Nanjing). Our data showed that PM2.5 increased the BCA level compared to saline treatment both in WT and TLR4 ${ }^{-/-}$mice (Fig. 2A). As expected, TLR4 KO mice showed decreased BCA level compared to wild type mice $(P=0.08$, Fig. 2A). Similarly, LDH showed exactly the same trend but more moderate level (Fig. 2B). These results indicated that, PM2.5 stimulation may initiate the immune response of the respiratory system in mice, especially in the lungs.

To determine whether PM2.5 treatment affects the expression of inflammatory cytokines in lung tissue, we collected lung tissue from the other side (the other part of the lung without lavage) and stored at $-80^{\circ} \mathrm{C}$. RNA was extracted before qRT-PCR was implemented. As shown in Fig. $2 \mathrm{C}$, among mice with the same background (either wild type mice or TLR4-/- mice), PM2.5 treatment increased the expression of inflammatory cytokines (HMGB1, TNFa, MCP-1/CCL2, CCR2) significantly compared to the saline treatment $(P<0.05)$. However, even though there is a trend that the expression of inflammatory cytokines could be attenuated in TLR4 knock out mice, only HMGB1 expression shows a statistical difference ( $P<$ 0.05). Therefore, our results revealed that, to some extent, TLR4 knock down can alleviate the inflammatory reaction of lung induced by PM2.5.

To determine whether PM2.5 could cause injury and inflammation of lung tissue, we also implemented approach of HE staining. After sacrifice the mice, the other part of the lung without lavage were collected and routinely fixed, embedded in paraffin, sectioned into $5 \mu \mathrm{m}$ in thickness and finally stained with haematoxylin and eosin (H-E). As shown in Fig. 2D, the lung tissues instilled with PM2.5 induced severe inflammatory damage, represented as infiltration of inflammatory cells (macrophages and lymphocytes) into airways and surrounding tissues. Although pulmonary inflammation in TLR4 knockout mice is also serious, it has been significantly improved. In summary, these results indicate that exposure to PM2.5 induces pronounced lung inflammation and TLR4 KO could alleviate the process of inflammation. 


\section{Liver inflammation}

To explore the liver inflammation caused by PM2.5 in mice, eyeball blood collection was committed after sacrifice the mice. Serum was separated by centrifugation at $2000 \mathrm{rpm}$ at $4^{\circ} \mathrm{C}$ after 15 minutes at room temperature. Serum was then stored at $-20^{\circ} \mathrm{C}$ and avoided from repeated freezing and thawing. BCA and some enzymes related to liver inflammation and liver injury (LDH, ALT, AKP) were measured in a short time according to the instructions provided by the manufacturer. LDH, Alanine Aminotransferase Kit (ALT) and Alkaline Phosphatase (AKP) were determined by the indicated kit (Jiancheng Bioengineering Institute, Nanjing). As shown in Fig. 3A, LDH, one of the markers for hepatocyte injury, significantly increased in PM2.5 treatment only in wild type mice, but not in TLR4 KO mice. AKP and ALT are the commonly used indicators for detecting liver inflammation. Our results showed that, PM2.5 exposure significantly increased AKP level still in wild type group (Fig. 3B). As to ALT level, there is a trend that it could be elevated by PM2.5 exposure in both groups, with no statistical significance due to high standard errors (Fig. 3C). Total protein concentration represented by BCA, showed similar trend with ALT (Fig. 3D). These results indicated that, PM2.5 exposure could induce liver injury and liver inflammation, and the TLR4, to some extent, could shorten the adverse effects on liver caused by PM2.5.

To determine whether PM2.5 exposure affects the expression of inflammatory cytokines in the liver, we collected liver tissue and stored at $-80^{\circ} \mathrm{C}$. RNA was extracted before qRT-PCR was implemented. Liver mRNA abundance was measured by qPCR and normalized to 36B4 levels. Similar to the lung tissue, PM2.5 stimulation resulted in robust induction of proinflammatory genes expression levels, and TLR4 KO could alleviate the progression of liver inflammation (Fig. 4A). No obvious pathologic alterations were found in the livers of all mice (Fig. 4B).

\section{Discussion}

Epidemiological investigation shows that, the level of PM2.5 in the air is positively related to the incidence and prevalence of respiratory inflammatory diseases (such as asthma), and can also lead to cardiovascular and other systemic diseases ${ }^{22,23}$. At present, the mechanism of haze (PM2.5) mainly includes local and systemic inflammatory response and oxidative stress. According to the existing investigation results, haze hazards are mainly manifested as acute effects, and its health effects are mainly manifested as acute respiratory inflammation, cardiovascular system disorder, and significant increase in the incidence and mortality of cardiopulmonary diseases ${ }^{24}$. The earliest health effect of the body after short-term exposure to pollutants ( 24 hours) is the activation of pulmonary inflammation and oxidative stress pathway 25 .

In addition to acting as a physical barrier against inhalation of toxic substances, respiratory epithelial cells can also participate in the occurrence and development of respiratory diseases through the release of inflammatory mediators. During the stimulation of harmful factors, airway epithelium and innate immune cells can release DAMP (damage related molecular model) molecules, and then activate the innate and acquired immunity of airway, releasing more inflammatory mediators. They interact with 
airway epithelium, and form a complex cytokine regulatory network, and trigger and promote the occurrence of asthma ${ }^{26}$.

It was found that TLR2 and TLR4 can bind to HMGB1 secreted by macrophages and neutrophils, which promotes NF-KB activation and induces inflammation. This indicated that TLR2 and TLR4 are the receptors of HMGB1. Recent studies have shown that HMGB1-DNA complex is not a single molecule, but a HMGB1-DNA complex ${ }^{27}$. Tian et al. confirmed that HMGB1-DNA complex can enter cells and activate intracellular receptors with the assistance of RAGE, thus activating TLR9 signaling pathway, promoting immune cell maturation and cell molecular secretion through TLR9 ${ }^{28}$. However, some experiments have confirmed that HMGB1-DNA can inhibit immune response or cause autophagy in some types of cells ${ }^{29}$. In addition, the activation pathway of rage mediated by HMGB1 is different from that of TLR4 mediated by HMGB1. The former only activated IKK $\beta$, while the latter activated IKKa and IKK $\beta$. Therefore, it can be seen that the signal transduction process of HMGB1 acting on cells is very complex, and it may activate cells to participate in related pathophysiological reactions in many ways.

Recently, when exploring the role of HMGB1-TLRs signaling pathway in ischemia-reperfusion injury, researchers found that human interference with the interaction of HMGB1-TLRs and blocking the activation of downstream signaling pathway can weaken the cascade reaction of inflammation and immune response triggered in the process of injury ${ }^{30-32}$. The previous work of our group found that TLR4 was involved in inducing the synthesis of TNFa in human macrophages by silica, and mediated the development of inflammation.

Hepatocytes can directly clear LPS from the bloodstream through hepatic uptake via TLR4, indicating their important role in inflammatory responses ${ }^{33}$. Both epidemiologic and controlled exposure studies in humans and animals have demonstrated an association between air pollution exposure and metabolic disorders such as diabetes ${ }^{34}$. It was also found that IL-6 levels were elevated in blood, liver, adipose tissue, and macrophages, which induced activation of the STAT3/SOCS3 pathway in liver ${ }^{35}$. Prenatal exposure to diesel exhaust PM (DEP) programmed the development of Non-alcoholic fatty liver disease (NAFLD) differently in the adult male offspring of mice fed normal chow and a high-fat diet, showing the pleotropic effects of exposure to adverse environmental factors in early life ${ }^{36}$.

Future studies are anticipated to make broad conceptual contributions, beyond the innate immunity field, to the understanding of essentials of cell biology.

\section{Conclusions}

In conclusion, we have identified that PM2.5 exposure could significantly induce both liver and lung inflammation and TLR4 knock out can alleviate the inflammatory reaction. Our findings suggest that, the activation of TLR signaling by PM2.5 plays an important role in lung and liver inflammatory response, which may result in activation of the innate and adaptive immunity. 


\section{Declarations}

\section{Ethics approval and consent to participate}

All the animal experiments were conducted following the protocols approved by the University Committee on the Use and Care of Animals (UCUCA).

\section{Consent for publication}

Not applicable.

\section{Availability of data and material}

The datasets generated during and/or analyzed during the current study are available from the corresponding author on reasonable request.

\section{Funding}

This study was supported by grants from National Natural Science Foundation of China (Grant No. 81602828, 81573112, 81703182, 31802024), Key R \& D and Promotion Projects of Henan Province (Grant No.182102311138), and Key Scientific Research Projects of Colleges and Universities of Henan Province (Grant No.15A330002), Training plan for Young Backbone Teachers in Universities of Henan Province (Grant/Award No. 2019GGJS151).

\section{Competing interests}

The authors declare that they have no competing interests.

\section{Authors' contributions}

Weidong Wu, Yang Liu: Conceptualization, review \& editing; Yang Liu, Bijie Jiang: Methodology, Software; Jianguo Lu, Congmin Li: Data curation, Formal analysis; Bijie Jiang: Writing - Original draft; Zhen An, Yichun Bai, Xinmei Zheng: Visualization, Investigation. Validation; Weidong Wu, Yang Liu, Bijie Jiang, Zhen An, Yichun Bai: Funding acquisition.

\section{Acknowledgements}


Not applicable.

\section{Abbreviations}

PM, particulate matter; TLRs, Toll-like receptors; BCA, protein concentration; LDH, Lactate Dehydrogenase ; HMGB1, High Mobility Group Box 1; TNFa, tumor necrosis factor alpha; MCP-1/CCL2, monocyte chemoattractant protein 1/ chemokine (C-C motif) ligand 2; CCR2, C-C chemokine receptor type 2; PM2.5, fine particulate matter ; AKP, Alkaline Phosphatase; PAMPs, pathogen associated molecular patterns; PRRs, pattern recognition receptors; DAMPs, damage-associated molecular patterns; TLR4, Toll Like Receptor 4; MyD88, myeloid differentiation factor 88; NF-kB, Nuclear Factor Kappa B; BALF, Bronchoalveolar Lavage Fluid; qPCR, Quantitative Real Time PCR; IKKa, ІКB kinase a; IKK $\beta$, ІкB kinase $\beta$; LPS, Lipopolysaccharides; STAT3, Signal transducer and activator of transcription 3; SOCS3, Suppressor of cytokine signaling 3; IL-6, Interleukin 6; NAFLD, Non-alcoholic fatty liver disease.

\section{References}

1. Gutierrez, M. de los A., Colman Lerner, J. E., Giuliani, D. S., Porta, A. A. \& Andrinolo, D. Comparative study of tear lipid composition in two human populations with different exposure to particulate matter in La Plata, Argentina. Environ. Sci. Pollut. Res.26, 6948-6956 (2019).

2. Li, X. et al. Air pollution during the winter period and respiratory tract microbial imbalance in a healthy young population in Northeastern China. Environ. Pollut.246, 972-979 (2019).

3. Park, M., Lee, J. S. \& Park, M. K. The Effects of Air Pollutants on the Prevalence of Common Ear, Nose, and Throat Diseases in South Korea: A National Population-Based Study. Clin. Exp. Otorhinolaryngol.12, 294-300 (2019).

4. Norbäck, D. et al. Home environment and health: Domestic risk factors for rhinitis, throat symptoms and non-respiratory symptoms among adults across China. Sci. Total Environ.681, 320-330 (2019).

5. Park, J. H., Oh, S. J. \& Lee, J. H. Effects of particulate matter on healthy human skin: a panel study using a smartphone application measuring daily skin condition. J. Eur. Acad. Dermatology Venereol.33, 1363-1368 (2019).

6. Katsuyama, Y. et al. 3-0-Laurylglyceryl ascorbate improves the development of sensitive skin through the reduction of oxidative stress. Exp. Dermatol.28, 64-68 (2019).

7. Hyun, Y. J. et al. Effect of fermented fish oil on fine particulate matter-induced skin aging. Mar. Drugs17, (2019).

8. Palm, N. W. \& Medzhitov, R. Pattern recognition receptors and control of adaptive immunity. Immunological Reviews vol. 227 221-233 (2009).

9. Takeuchi, O. \& Akira, S. Pattern Recognition Receptors and Inflammation. Cell vol. 140 805-820 (2010).

10. Geijtenbeek, T. B. H. \& Gringhuis, S. I. Signalling through C-type lectin receptors: Shaping immune responses. Nature Reviews Immunology vol. 9 465-479 (2009). 
11. Ishikawa, H. \& Barber, G. N. STING is an endoplasmic reticulum adaptor that facilitates innate immune signalling. Nature455, 674-678 (2008).

12. Saber, T. et al. Toll-like receptor 2 induced angiogenesis and invasion is mediated through the tie2 signalling pathway in rheumatoid arthritis. PLoS One6, (2011).

13. Kowalski, M. L. et al. Increased responsiveness to Toll-like receptor 4 stimulation in peripheral blood mononuclear cells from patients with recent onset rheumatoid arthritis. Mediators Inflamm.2008, (2008).

14. Dange, R. B., Agarwal, D., Teruyama, R. \& Francis, J. Toll-like receptor 4 inhibition within the paraventricular nucleus attenuates blood pressure and inflammatory response in a genetic model of hypertension. J. Neuroinflammation12, (2015).

15. Long, T. et al. Polygonatum sibiricum polysaccharides play anti-cancer effect through TLR4MAPK/NF-kB signaling pathways. Int. J. Biol. Macromol.111, 813-821 (2018).

16. Ma, Z. et al. Satellite-based spatiotemporal trends in PM2.5 concentrations: China, 2004-2013. Environ. Health Perspect.124, 184-192 (2016).

17. Van Donkelaar, A., Martin, R. V., Brauer, M. \& Boys, B. L. Use of satellite observations for long-term exposure assessment of global concentrations of fine particulate matter. Environ. Health Perspect.123, 135-143 (2015).

18. Geng, J. et al. PM2.5 promotes plaque vulnerability at different stages of atherosclerosis and the formation of foam cells via TLR4/MyD88/NFkB pathway. Ecotoxicol. Environ. Saf.176, 76-84 (2019).

19. Qiu, Y. N. et al. PM2.5 induces liver fibrosis via triggering ROS-mediated mitophagy. Ecotoxicol. Environ. Saf.167, 178-187 (2019).

20. Jeong, S. et al. PM2.5 Exposure in the Respiratory System Induces Distinct Inflammatory Signaling in the Lung and the Liver of Mice. J. Immunol. Res.2019, (2019).

21. Zheng, Z. et al. Exposure to fine airborne particulate matters induces hepatic fibrosis in murine models. J. Hepatol.63, 1397-1404 (2015).

22. Scheers, H., Jacobs, L., Casas, L., Nemery, B. \& Nawrot, T. S. Long-Term Exposure to Particulate Matter Air Pollution Is a Risk Factor for Stroke: Meta-Analytical Evidence. Stroke46, 3058-3066 (2015).

23. Loomis, D., Huang, W. \& Chen, G. The International Agency for Research on Cancer (IARC) evaluation of the carcinogenicity of outdoor air pollution: Focus on China. Chinese Journal of Cancervol. 33 189-196 (2014).

24. Lu, F. et al. Systematic review and meta-analysis of the adverse health effects of ambient PM2.5 and PM10 pollution in the Chinese population. Environmental Research vol. 136 196-204 (2015).

25. Roy, A. et al. The cardiopulmonary effects of ambient air pollution and mechanistic pathways: A comparative hierarchical pathway analysis. PLoS One9, (2014). 
26. Ullah, M. A. et al. Receptor for advanced glycation end products and its ligand high-mobility group box-1 mediate allergic airway sensitization and airway inflammation. J. Allergy Clin. Immunol.134, (2014).

27. Yu, M. et al. HMGB1 signals through toll-like receptor (TLR) 4 and TLR2. Shock26, 174-179 (2006).

28. Tian, J. et al. Toll-like receptor 9-dependent activation by DNA-containing immune complexes is mediated by HMGB1 and RAGE. Nat. Immunol.8, 487-496 (2007).

29. Liu, L. et al. HMGB1-DNA complex-induced autophagy limits AIM2 inflammasome activation through RAGE. Biochem. Biophys. Res. Commun.450, 851-856 (2014).

30. Mcdonald, K. A. et al. Toll-like receptor 4 (TLR4) antagonist eritoran tetrasodium attenuates liver ischemia and reperfusion injury through inhibition of high-mobility group box protein B1 (HMGB1) signaling. Mol. Med.20, 639-648 (2014).

31. Wang, J. et al. TLR4-HMGB1-, MyD88- and TRIF-dependent signaling in mouse intestinal ischemia/reperfusion injury. World J. Gastroenterol.21, 8314-8325 (2015).

32. Zhu, Z. \& Fang, Z. Statin protects endothelial cell against ischemia reperfusion injury through HMGB1/TLR4 pathway. Int. J. Cardiol.203, 74 (2016).

33. Yang, D. et al. Kukoamine B promotes TLR4-independent lipopolysaccharide uptake in murine hepatocytes. Oncotarget7, 57498-57513 (2016).

34. Reyes-Caballero, H. et al. Air pollution-derived particulate matter dysregulates hepatic Krebs cycle, glucose and lipid metabolism in mice. Sci. Rep.9, (2019).

35. Long, M. hui et al. PM2.5 aggravates diabetes via the systemically activated IL-6-mediated STAT3/SOCS3 pathway in rats' liver. Environ. Pollut.256, (2020).

36. Wang, X. et al. Prenatal exposure to diesel exhaust PM2.5 programmed non-alcoholic fatty liver disease differently in adult male offspring of mice fed normal chow and a high-fat diet. Environ. Pollut.255, (2019).

\section{Figures}




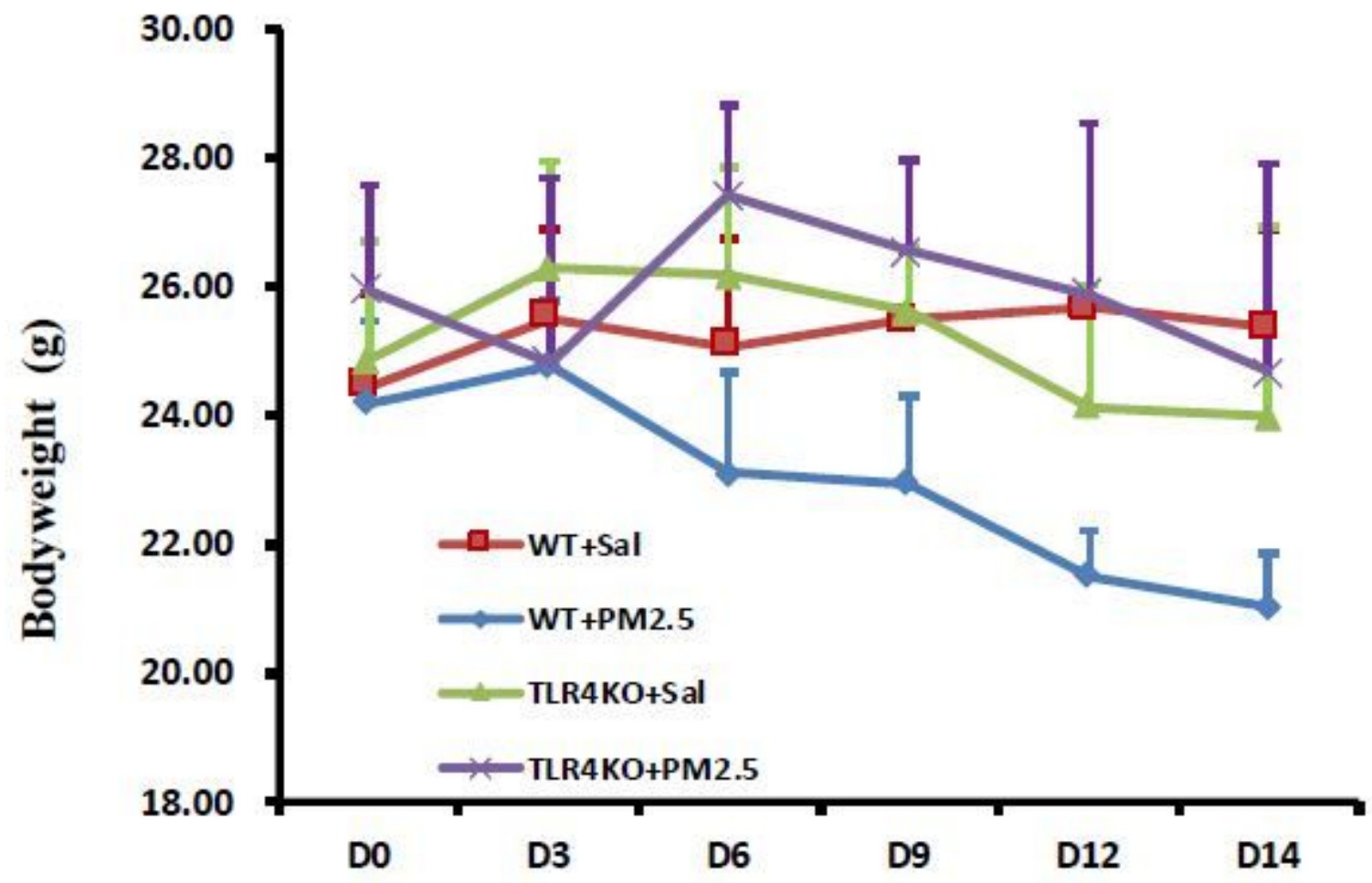

Figure 1

Body weight changes of mice during the whole experiment. 

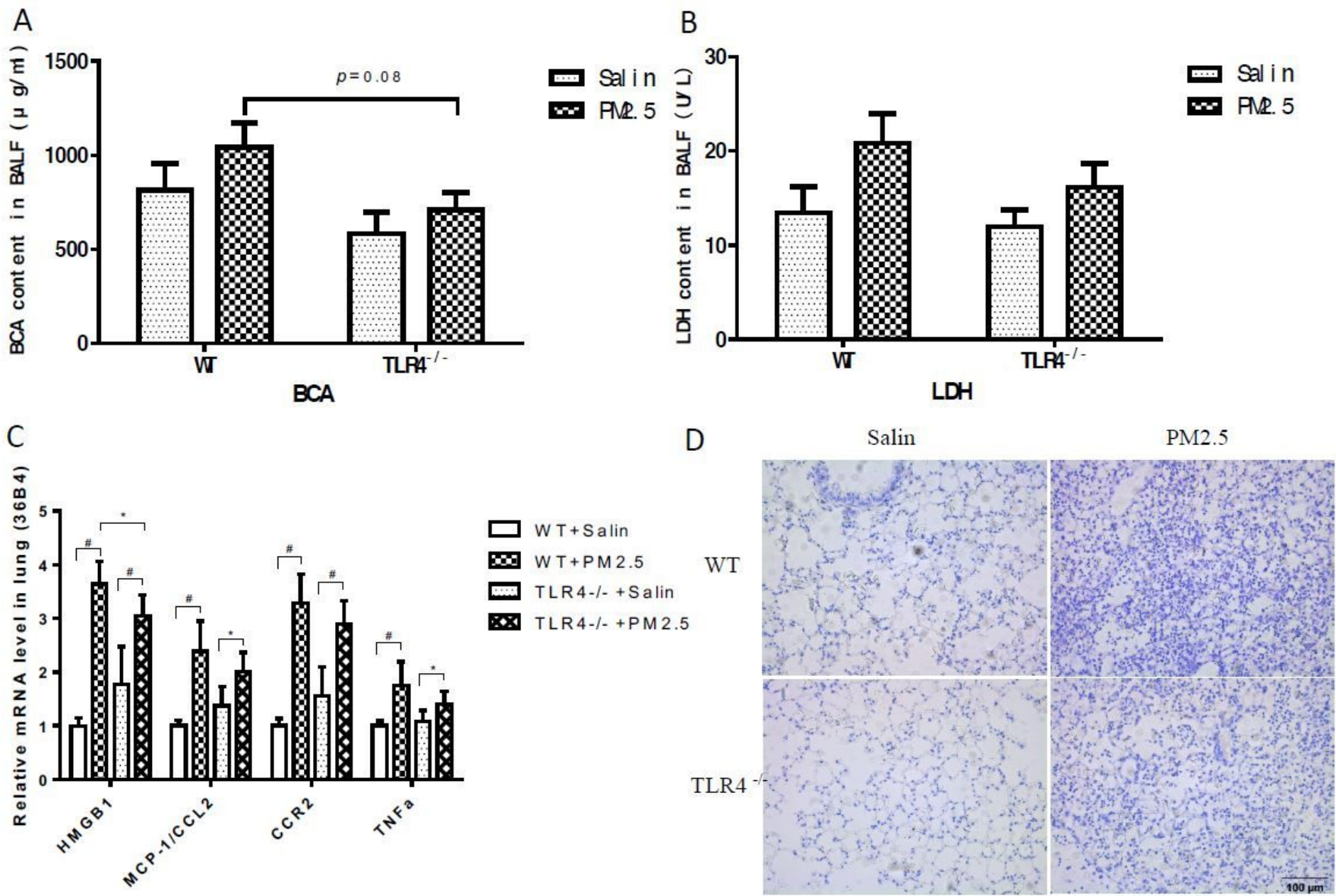

Figure 2

TLR4-/- decreased lung inflammation caused by PM2.5. 
A

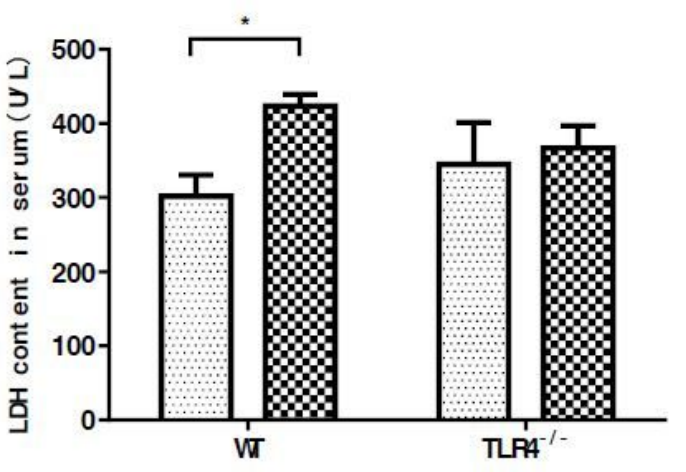

C

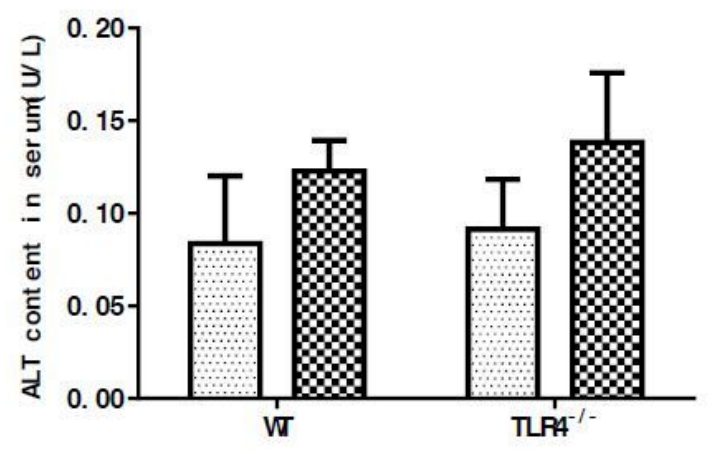

Sal in

PNR. 5
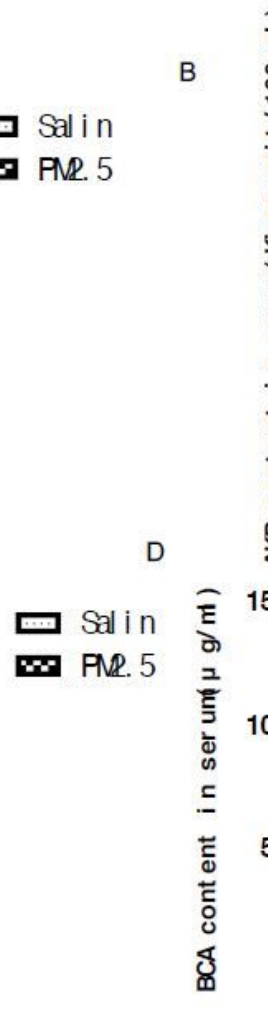

E

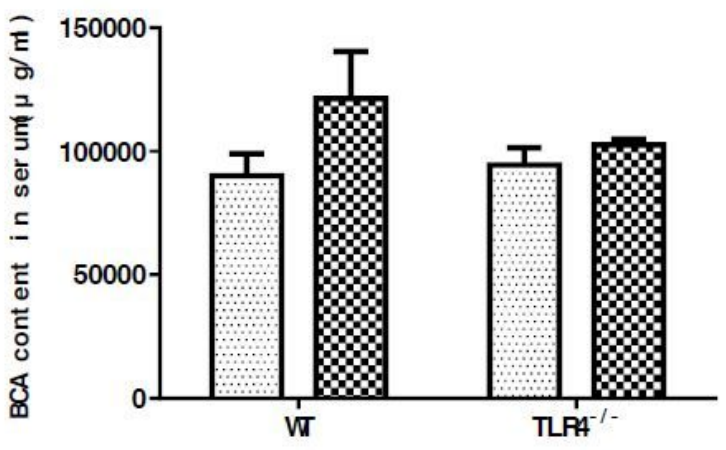

Figure 3

TLR4 could decrease the adverse effects on liver caused by PM2.5.

A

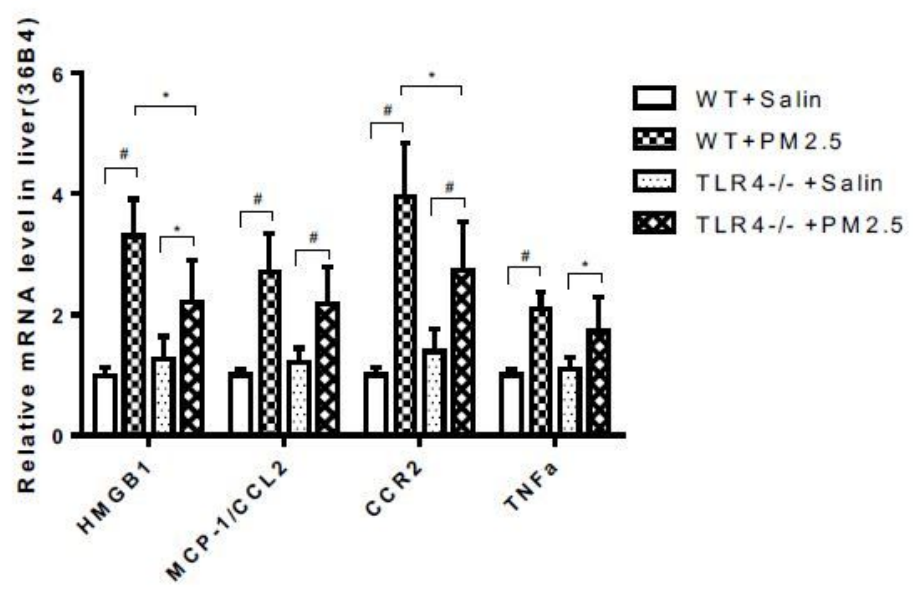

B

Salin

Sal in

m PNR. 5

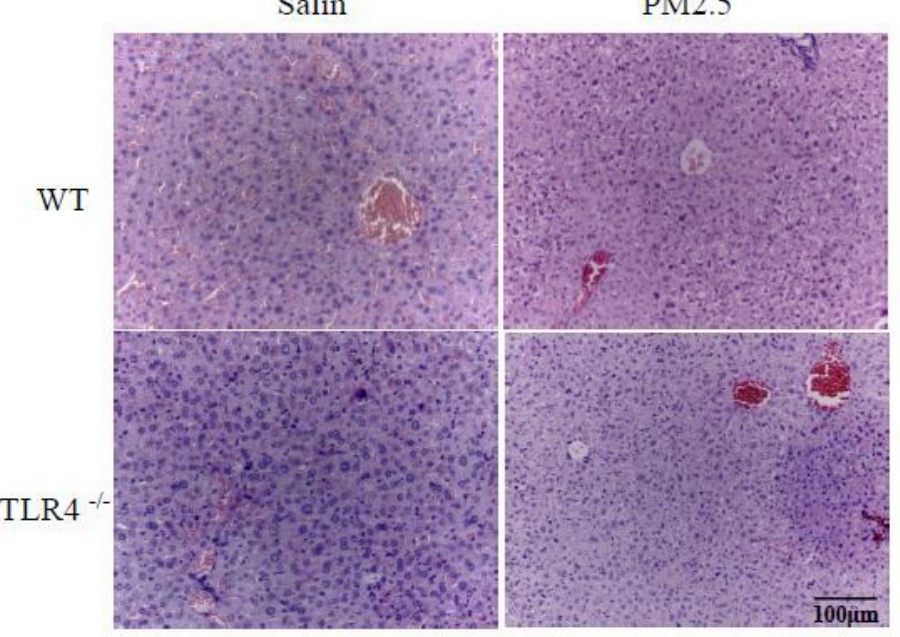

Figure 4

TLR4 KO could alleviate the progression of liver inflammation.

Supplementary Files 
This is a list of supplementary files associated with this preprint. Click to download.

- coverletter20210401.docx

- graphicalabstract.pdf 